\title{
Autologous Platelet-Rich Plasma to Prevent a Thin Endometrium in Patients Undergoing Clomiphene Citrate Therapy : A Pilot Prospective Self-Controlled Trial
} Original

Article

\author{
Ahmed Walid Anwar Morad, Hatem Elgendy, Tamer Mahmoud Assar \\ Department of Obstetrics and Gynecology, Faculty of Medicine, Benha University, Egypt
}

\begin{abstract}
Aim: This is a pilot prospective self-controlled trial study. It aimed to explore the efficacy of intrauterine infusion of autologous platelet-rich plasma (PRP) in infertile ladies as an adjuvant to clomiphene citrate (CC) in ladies with previous CC failure attributed to a thin endometrium $(\leq 7 \mathrm{~mm})$.

Materials and Methods: This study included thirty anovulatory PCOS women with CC failure for 3 cycles with a thin endometrium $(7 \mathrm{~mm}$ ). All patients underwent ovarian stimulations for 2 cycles; control cycle (30 women received CC alone and one got pregnant) and study cycle (29 women received CC plus an intrauterine infusion of PRP). Main endpoints included the comparison of the endometrial thickness (ET) and endometrial vascularity pattern by power Doppler on the day of hCG injection between both treatment cycles.

Results: There was a statistically significant increase in the endometrial thickness on the day of hCG injection with $\mathrm{CC}$ plus PRP compared to CC alone $(8.98 \pm 1.31 \mathrm{vs,} 5.8 \pm 1.2 ; p<0.0001)$. There was a significant improvement in the endometrial blood flow with the use of PRP $(p=0.00004)$. The pregnancy rate is significantly higher in the PRP cycles compared to control cycles (7 pregnancies $(24.14 \%)$ vs. one pregnancy $(3.3 \%)$ respectively; $p=0.0257)$.

Conclusion: Intrauterine infusion PRP seems to be a promising adjuvant to increase endometrial thickness and endometrial blood flow with subsequent improvement in clinical pregnancy rate in ladies with CC failure due to thin endometrium.
\end{abstract}

Key Words: Autologous platelet-rich plasma, clomiphene citrate, PCOS, thin endometrium

Received: 11 October 2020, Accepted: 06 December 2020

Corresponding Author: Ahmed Walid Anwar Morad, Department of Obstetrics and Gynecology, Faculty of Medicine, Benha University, Egypt, Tel.: +201224214435, E-mail: awalid217@fmed.bu.edu.eg

ISSN: 2090-7265, February 2021, Vol.11, No. 1

\section{INTRODUCTION}

Anovulation represents $20 \%$ of all infertility cases $^{[1]}$. Normogonadotropic normoestrogenic anovulation including polycystic ovarian syndrome $(\mathrm{PCOS})^{[2]}$, classified according to the World Health Organization (WHO) as type-2 anovulation constitutes around 85\% of anovulatory infertility ${ }^{[3]}$. Clomiphene citrate (CC) is the first-choice ovulation induction agent in women with WHO type-2 anovulatory infertility ${ }^{[2,3]}$. The discordance between the higher ovulation rate $(60-85 \%)$ and the lower pregnancy rate $(30-40 \%)$ in the CC-stimulated cycles may be assigned to the $\mathrm{CC}$ antiestrogenic effects on the endometrium and the cervical mucus ${ }^{[4]}$.

Someauthors indicated thatCC through its antiestrogenic effects has a negative impact on the endometrial receptivity, as it reduces endometrial development and uterine blood flow manifested as a thin endometrium in $15-50 \%$ of cases with consequent implantation failure and early pregnancy $\operatorname{loss}^{[1,5]}$. Thin endometrium constitutes a major cause of $\mathrm{CC}$ failure where women do not get pregnant despite the occurrence of ovulation with $\mathrm{CC}^{[6]}$. Therefore, subsequent step therapy for such patients is the gonadotropin therapy that carries the potential risks of multiple gestations or ovarian hyperstimulation syndrome (OHSS) $)^{[7]}$. Similarly, laparoscopic ovarian drilling is associated with possible destruction to ovarian reserve and potential adhesion development ${ }^{[8]}$. Other options include earlier starting of $\mathrm{CC}$, the use of aromatase inhibitors for ovarian stimulation, and delaying human chorionic gonadotropin (hCG) administration. Different adjuvant therapies have been tested to increase the ET including systemic or vaginal estrogen, low dose Aspirin, vaginal Sildenafil, vitamin E, Pentoxifylline, electroacupuncture and intrauterine infusion of granulocyte-colony stimulating factor (G-CSF).

Unfortunately, the improving effect of these options on the ET in women under controlled ovarian hyperstimulation $(\mathrm{COH})$ or those receiving $\mathrm{CC}$ is controversial ${ }^{[9,10]}$. Platelet rich plasma is a blood plasma fraction prepared from the fresh whole blood that has been enriched with platelets and several growth factors and cytokines. Chang et al. 2015 suggested intrauterine instillation of PRP as a novel technique for treating the thin endometrium in women under $\mathrm{COH}$ with promising results ${ }^{[11]}$. 


\section{AIM OF THE WORK}

The current study was done to assess the efficacy of intrauterine instillation of PRP in increasing the endometrial thickness in infertile women with clomiphene citrate failure due to the thin endometrium.

\section{PATIENTS AND METHODS}

The protocol of this pilot prospective selfcontrolled trial was approved by the Local Ethics Board of the Obstetrics and Gynecology Department, Benha University, and recorded at Clinical Trials gov: NCT03770026. Signed well-counseled consents obtained from selected cases before the study start. The study involved 30 women with primary infertility for $\geq 2$ years with anovulatory PCOS diagnosed based on the Rotterdam criteria (2003 ESHRE/ASRM consensus) ${ }^{[12]}$, aged $18-35$ years, early follicular phase serum FSH $<10 \mathrm{mIU} / \mathrm{mL}$ confirmed bilateral tubal patency by hysterosalpingography or laparoscopy and her husband semen analysis satisfied the modified WHO criteria ${ }^{[13]}$. All participants already had CC failure due to the thin endometrium (i.e., $<7 \mathrm{~mm}$ on the day of hCG injection) for 3 CC-stimulation cycles. Clomiphene citrate failure was defined as the failure to achieve pregnancy with $\mathrm{CC}$ induction in a dose of $100 \mathrm{mg} /$ day from $3^{\text {rd }}$ to $7^{\text {th }}$ day of the cycle, despite adequate ovulation documented by sonographic imaging and mid-luteal serum progesterone $\geq 3$ $\mathrm{ng} / \mathrm{ml}^{[14]}$. Women with previous ovarian cystectomy or laparoscopic ovarian diathermy, endocrine disorders as hyperprolactinemia, congenital adrenal hyperplasia, thyroid function abnormalities and clinically speculated Cushing's syndrome, pelvic pathologies as uterine leiomyoma, adenomyosis, congenital uterine malformation, or ovarian tumors, chronic hepatic, cardiovascular, or renal disease and those using gonadotropins, or hormonal contraception during the latest 6 months were not involved in the study.

Patients underwent two ovarian stimulation cycles. The control cycle (CC only cycle); registered cases (30 women) received $\mathrm{CC}$ induction (Clomid 50 mg tab; Aventis pharma S.AE, Global Napi Pharmaceuticals, Cairo, Egypt) at a dose of $100 \mathrm{mg} /$ day for 5 days starting from cycle day 3. Beginning from the $9^{\text {th }}$ day of the cycle, follicle growth monitoring by transvaginal sonography was performed every other day. When the mean diameter of the leading follicle reached $18 \mathrm{~mm}, 10000$ IU of highly purified hCG (Choriomon 5000 IU Vial; IBSA, Switzerland) was injected intramuscularly for ovulation triggering followed by timed intercourse 36 hours later.

Two weeks later serum $\beta$ subunit hCG was tested for the diagnosis of biochemical pregnancy. Cervical flushing with $1 \mathrm{ml}$ of $0.9 \%$ normal saline was done in the control group at cycle day 8 and repeated at cycle day 10 to guarantee patient-blinded intervention. Study cycle (CC + intrauterine PRP); women who didn't get pregnant within the control cycle ( 29 women) were ordered to have an additional cycle of $\mathrm{CC}$ induction at the same regimen, in addition to the intrauterine infusion of PRP performed at cycle day 8 and at cycle day 10 .

No estradiol or any additional adjuvants were used in either the control or the study cycle apart from those mentioned above. Autologous platelet-rich plasma (PRP); on the $8^{\text {th }}$ day of the cycle, $15 \mathrm{~cm} 3$ of the patient's venous blood was withdrawn in a syringe containing $5 \mathrm{ml}$ of anticoagulant (ACD-A) followed by 10 -min centrifugation at $200 \times \mathrm{g}$. The blood was split into three fractions; the bottom fraction containing blood cells, a supernatant fraction containing the cellular plasma and a buffy coat fraction in between. The plasma and buffy coat fractions were re-centrifuged in another tube at $500 \times \mathrm{g}$ for $10-\mathrm{min}$. The formed platelet pellet was mixed with $1 \mathrm{~cm} 3$ of the supernatant; therefore, $0.5-1 \mathrm{ml}$ PRP was collected. Under aseptic precautions and ultrasound guidance, PRP $(0.5-1 \mathrm{ml})$ was immediately instilled into the uterine cavity using an IUI catheter ${ }^{[1]}$.

Evaluation of the study endpoints: During the control and the study cycles, the endometrial thickness and uterine Doppler study were assessed using a $7.5 \mathrm{MHz}$ vaginal probe with a Doppler facility (Voluson 730 PRO V, GE Healthcare, USA). Ultrasound examinations were done by the same sonographer to avoid interobserver variability. The participant was examined with an empty bladder between 8.00 and 10.00 A.M. to eliminate the influences of circadian rhythmicity on the uterine perfusion and relaxed for $20 \mathrm{~min}$ before the Doppler examination to lessen the influences of exercises on uterine Doppler indices. Endometrial thickness was measured in the midsagittal plane as the greatest distance between the two endometrial-myometrial interphase in the fundal region. It is measured twice and the mean was recorded. Applying Power Doppler with machine adaption for endometrium, vascular signals were specified. Endometrial vascularity was evaluated in four zones; zone 1 vascularity described blood vessels reached the hypoechoic endometrial-myometrial junction, zone 2 vascularity described blood vessels reached the outer hyperechoic line of the endometrium, zone 3 vascularity described blood vessels reached the intervening hypoechoic area and zone 4 vascularity described blood vessels reached the central echogenic line. Endometrial vascularity was classified according to the power Doppler signals into excellent vascularity by the presence of at least five signals in zones 3 and 4 , modest vascularity with up to 4 signals reaching zones 3 and 4 , and poor vascularity with less than 
one signal in zones 3 and $4^{[15]}$. The primary endpoints were the endometrial thickness, and power Doppler evaluation of the endometrial vascularity on the day of hCG injection, however; the second endpoint was the clinical pregnancy rate. Clinical pregnancy was affirmed by sonographic detection of an intrauterine gestational sac with fetal heartbeats at 6-weeks' gestation.

\section{STATISTICAL ANALYSIS:}

The obtained data were statistically explored with The Statistical Package for Social Sciences (SPSS, Chicago, USA) software version 17.0. Numerical information was represented as the mean \pm the standard deviation, and the difference was tested via the paired Student t-test. Categorical data were stated as numbers and percentages and the difference was tested using the Chi-square test and Fisher exact test as appropriate. A p-value of less than 0.05 .was stated as statistically significant.

\section{RESULTS}

We selected 30 infertile women with $\mathrm{CC}$ failure (ovulatory cycles, with no pregnancy) and thin endometrium (less than $7 \mathrm{~mm}$ ) in at least three of the $\mathrm{CC}$-stimulated cycles. During the control (CC alone) cycle pregnancy occurred in one case; therefore, 29 women experienced the study cycle intervention with intrauterine PRP as an adjuvant treatment with the standard CC therapy. The demographic, clinical, and laboratory characteristics of the participants at the time of study enrollment are described in Table 1.There was a statistically significant increase in the endometrial thickness on the day of hCG injection with $\mathrm{CC}$ plus PRP treatment compared to $\mathrm{CC}$ only treatment $(8.98 \pm 1.31$ vs $5.8 \pm 1.2 ; p<0.0001)$. However, 4 women in our study had endometrial thickness less than $7 \mathrm{~mm}$ despite the use of PRP. There was a significant improvement in the endometrial blood flow with the use of PRP $(p=0.00004)$. Eight women demonstrating pre-PRP instillation poor to modest vascularity had an excellent post-PRP instillation vascularity pattern; whereas, in 17 patients, the vascularity pattern enhanced to modest from poor. Only four cases persisted to have a poor vascularity pattern. The pregnancy rate is significantly higher in the PRP cycles compared to the control cycles; seven pregnancies $(24.14 \%)$ vs. one pregnancy $(3.3 \%)$, respectively; $p=0.0257$ (Table 2 ).

No significant difference $(p>0.05)$ was noted between the control and the study cycles concerning the number of the leading follicles $>18 \mathrm{~mm}$ in diameter on the day of hCG injection and the number of cycle days till hCG injection (Table 2).

Table 1: Characteristic data of the participants at the time of study enrollment

\begin{tabular}{lc}
\hline Variable & Value \\
\hline Age (years) & $22.8 \pm 3.2$ \\
BMI (kg/m2) & $25.5 \pm 3.1$ \\
Duration of infertility (years) & $2.9 \pm 0.7$ \\
Hormonal profile & \\
FSH (mIU/ml & $5.6 \pm 1.84$ \\
LH (mIU/ml) & $7.83 \pm 2.78$ \\
Midcycle E2 (pg/ml) & $471.1 \pm 72.0$ \\
Midluteal progesterone (ng/ml) & $15.5 \pm 5.3$ \\
\hline
\end{tabular}

Data are shown as mean $\pm \mathrm{SD}$, ranges, numbers and percentage.

BMI, body mass index; kg/m2, kilogram per square meter; FSH, Follicle-stimulating hormone; LH, Luteinizing hormone; E2, Estradiol; pg/ $\mathrm{ml}$, picogram/milliliter; $\mathrm{mIU} / \mathrm{ml}$, Milli-International Units per Milliliter 
Table 2: Cycle characteristics and pregnancy outcome in the control (CC alone) and the study (CC + platelet-rich plasma) cycles of treatment

\begin{tabular}{|c|c|c|c|}
\hline Variables & Control Cycle $(n=30)$ & PRP Cycle $(n=29)$ & p-value \\
\hline Number of follicle $>18 \mathrm{~mm}$ & $2.3 \pm 0.5$ & $2.13 \pm 0.12$ & 0.0802 \\
\hline $\begin{array}{l}\text { Number of follicles P18 } \\
\mathrm{mm} \text { in diameter }\end{array}$ & $2.47 \pm 1.19(1-4)$ & $2.27 \pm 1.09(1-3)$ & 0.5040 \\
\hline Days until hCG administration & $12.0 \pm 1.8$ & $11.9 \pm 1.7$ & 0.8273 \\
\hline $\begin{array}{l}\text { Endometrial thickness (mm) } \\
\text { on the day of FSH injection }\end{array}$ & $5.8 \pm 1.2(4.5-6.9)$ & $8.98 \pm 1.31(5.8-10.4)$ & $<0.0001$ \\
\hline \multicolumn{4}{|l|}{$\begin{array}{l}\text { Power Doppler vascularity } \\
\text { pattern of the endometrium }\end{array}$} \\
\hline Poor & $21(70 \%)$ & $4(13.8 \%)$ & \\
\hline Modest & $8(26.7 \%)$ & $17(58.6 \%)$ & 0.00004 \\
\hline Excellent & $1(3.3 \%)$ & $8(27.6)$ & \\
\hline Clinical pregnancy per cycle & $1(3.3 \%)$ & $7(24.14 \%)$ & 0.0257 \\
\hline
\end{tabular}

Data are presented as means $\pm \mathrm{SD}$, or number (Percent)

\section{DISCUSSION}

The thin endometrium is the most frequent unintended anti-estrogenic consequence of the conventional CC treatment for ovarian stimulation. It represents one of the major causes of CC failure ${ }^{[3,6]}$. It is not precisely known how a thin endometrium reduces the prospect of conception, but it is assumed that the closeness of the embryo to the basal endometrial layer with rich reactive oxygen species could be harmful to embryonic development and implantation, or probably that with a thin endometrium, there is a non-enough soil to retain the seed ${ }^{[17]}$. In the present study, we investigated the effects of PRP intrauterine infusion as CC-adjuvant on the endometrial thickness in PCOS women with $\mathrm{CC}$ failure due to thin endometrium. In the current study, we defined thin endometrium as the endometrium of less than $7 \mathrm{~mm}$ thickness. This definition is founded on evidence derived from the ovum donation model. Where, following the endometrium preparation with estrogen replacement, the endometrial biopsy exhibited maturation arrest with an endometrial thickness of $<7 \mathrm{~mm}$. Nevertheless, an in-phase endometrium was found with an endometrial thickness of $\geq 7 \mathrm{~mm}^{[18]}$.

Although a $7 \mathrm{~mm}$ endometrial thickness is widely accepted as a minimum endometrial thickness acceptable for conception; yet, this cutoff is not crucial, as pregnancies can exist at lower cutoffs ${ }^{[19]}$. Unfortunately, there are a limited number of studies on the use of PRP in the field of infertility. The majority of these studies evaluated the impact of PRP on poor endometrium of IVF cycles $^{[20]}$.The current study revealed that PRP when added as an adjuvant to $\mathrm{CC}$ increased endometrial thickness and enhanced endometrial blood flow significantly. We have also reported a significant rise in the clinical pregnancy rate. These findings are in agreement with Sipahi, who assessed the influence of PRP on the endometrium in women with unexplained infertility undergoing insemination. He reported that PRP intrauterine instillation increases the ET significantly in women having an insufficient endometrial thickness with CC. However, no significant increase was reported in the clinical pregnancy rate $^{[21]}$. Also, the findings of the current study are in agreement with the former studies in assisted reproduction (summarized in Table 3). Those ensuring that the local instillation of PRP is significantly effective in increasing ET, endometrial vascularity and pregnancy rates of infertile women with a thin endometrium or repeated implantation failure ${ }^{[11,22,23,24]}$ Sami et al. 2020 in their review article that includes mainly case reports, case series, letters, and small sample-sized pilot studies reported the efficiency of local infusion of PRP in successful implantation in IVF-Cycles. They recommended that additional studies with a large 
number of patients should be done to explore more protocols in poor endometrium management ${ }^{[20]}$. The beneficial effects of PRP in these patients may be explained by its ability to enhance the endometrial receptivity and vascularity as it holds various growth factors and cytokines such as platelet-derived growth factor, fibroblast growth factor, transforming growth factor, vascular endothelial growth factor, insulin-like growth factor I-II, connective tissue growth factor and IL-8 the that have positive effects on local tissue repair and endometrial receptivity ${ }^{[1,20,25]}$. In the current study, 4 women had a persistent endometrial thickness of less than $7 \mathrm{~mm}$ despite the use of PRP, therefore; further investigations are mandatory to identify the cause of thin endometrium ${ }^{[19,20]}$.
This study has some limitations; small sample size, the endometrium of the natural cycle was not studied and the endometrial growth is a dynamic phenomenon as it continues after the time of hCG injection so it is wise to repeat the measures in the mid-luteal phase. However, these results are of interest as this study has the following advantages; a pilot study evaluated the impact of PRP on poorly developed endometrium in non-IVF cycles. The prospective self-controlled design of the study has the advantage of eliminating the confounding effects of demographic and clinical characteristic factors as each case is the control for itself. PRP is simply obtained, low-cost, and safe preparation as it is extracted from the patient's own blood.

Table 3: Summary of studies evaluating autologous platelet-rich plasma (PRP) infusion in infertile cases with thin endometrium

\begin{tabular}{|c|c|c|c|c|}
\hline Author, year & Study design & Population & $\begin{array}{l}\text { Sample } \\
\text { size }\end{array}$ & outcome \\
\hline Chang et al. $(2015)^{[11]}$ & Prospective Cohort study & $\begin{array}{l}\text { Thin endometrial } \\
(<7 \mathrm{~mm}) \text { to the } \\
\text { standard hormonal } \\
\text { preparation of FET }\end{array}$ & 5 & $\begin{array}{l}\text { Endometrial thickness } \\
\text { (ET) increased in } 100 \% \\
\text { Pregnancy rate } 80 \%\end{array}$ \\
\hline Tandulwadkar et al. (2017) ${ }^{[22]}$ & Prospective Cohort study & $\begin{array}{l}\text { Recurrent implantation } \\
\text { failure due to poor } \\
\text { endometrial lining. }\end{array}$ & 68 & $\begin{array}{c}\text { ET and vascularity } \\
\text { increased post- PRP. } \\
\text { Clinical pregnancy }(45.1 \%) \text {. }\end{array}$ \\
\hline Eftekhar et al. $(2018)^{[23]}$ & Randomized clinical trial & $\begin{array}{l}\text { Thin endometrial } \\
(<7 \mathrm{~mm}) \text { to the } \\
\text { standard hormonal } \\
\text { preparation of FET }\end{array}$ & $\begin{array}{l}40 \text { in } \\
\text { PRP } 43 \text { in } \\
\text { control }\end{array}$ & $\begin{array}{l}\text { PRP higher ET }(0.001), \\
\text { implantation } \\
(\mathrm{P}=0.002) \text { and pregnancy } \\
\text { rate }(\mathrm{P}=0.044) .\end{array}$ \\
\hline Chang et al. $(2019)^{[24]}$ & $\begin{array}{l}\text { Prospective } \\
\text { interventional study }\end{array}$ & $\begin{array}{c}\text { Patients with thin } \\
\text { endometrium }<7 \mathrm{~mm} \\
\text { and prior canceled FET. }\end{array}$ & $\begin{array}{c}34 \text { in PRP } \\
30 \text { in } \\
\text { control }\end{array}$ & $\begin{array}{l}\text { PRP had higher ET and } \\
\text { lower cancellation rate } \\
\text { Higher implantation and clinical } \\
\text { pregnancy rate in favor of PRP }\end{array}$ \\
\hline Sipahi $(2019)^{[21]}$ & $\begin{array}{l}\text { Prospective } \\
\text { interventional study }\end{array}$ & $\begin{array}{l}\text { Patients with } \\
\text { CC induced thin } \\
\text { endometrium }(<7 \\
\text { mm) underwent IUI }\end{array}$ & $\begin{array}{c}12 \text { in PRP } \\
12 \text { in } \\
\text { control }\end{array}$ & $\begin{array}{l}\text { PRP had significant higher ET and } \\
\text { non-significant difference in CPR }\end{array}$ \\
\hline
\end{tabular}

IUI; Intrauterine Insemination, CPR; Clinical pregnancy rate, FET; Fresh embryo transfer 


\section{CONCLUSION}

Autologous platelet-rich plasma significantly increased endometrial thickness, endometrial blood flow with non-significant increased pregnancy rate in anovulatory PCOS patients with clomiphene citrate failure due to thin endometrium. Randomized trials of larger sample sizes are recommended to approve these conclusions.

\section{CONFLICT OF INTEREST}

There are no conflicts of interests.

\section{REFERENCES}

1. Weiss NS, Braam S, König TE et al. How long should we continue clomephine citrate in anovulatory women? Hum Reprod. 2014;29(11):2482-6.

2. Brown J, Farquhar C, Beck J et al. Clomiphene and antioestrogens for ovulation induction in PCOS. Cochrane Database of Syst Rev. 2009; 4:CD002249.

3. The ESHRE Capri Workshop Group. Health and fertility in World Health Organization type 2 anovulatory women. Hum Reprod Update. 2012; 18:586-99.

4. Homburg R. Clomiphene citrate-end of an era? A mini-review. Hum Reprod 2005; 20:2043-51.

5. Thessaloniki ESHRE/ASRM-sponsored PCOS Consensus Workshop Group. Consensus on infertility treatment related to polycystic ovary syndrome. Hum Reprod 2008; 23(1):462-77.

6. Balen AH. Ovulation induction in the management of anovulatory polycystic ovary syndrome. Mol Cell Endocrinol. 2013; 373:77-82.

7. Homburg R, Hendriks ML, König TE et al. Clomifene citrate or low-dose FSH for the firstline treatment of infertile women with anovulation associated with polycystic ovary syndrome: a prospective randomized multinational study. Hum Reprod. 2012; 27:468-73.

8. Fernandez H, Morin-Surruca M, Torre A et al. Ovarian drilling for surgical treatment of polycystic ovarian syndrome: a comprehensive review. Reprod Biomed Online. 2011; 22(6):556-68
9. Eftekhar M, Tabibnejad N, Tabatabaie AA. The thin endometrium in assisted reproductive technology: An ongoing challenge. MEFSJ 2018; 23(1): 1-7

10. Sharma S10-Rani G, Bose G, Saha I, Bathwal $\mathrm{S}$, Chakravarty BN. Tamoxifen is Better than Low-Dose Clomiphene or Gonadotropins in Women with Thin Endometrium $(<7 \mathrm{~mm})$ after Clomiphene in Intrauterine Insemination Cycles: A Prospective Study. J Hum Reprod Sci. 2018 JanMar;11(1):34-39.

11. Chang Y11-Li J, Chen Y, Wei L, Yang X, Shi $\mathrm{Y}$, Liang $\mathrm{X}$. Autologous platelet-rich plasma promotes endometrial growth and improves pregnancy outcome during in vitro fertilization. Int J Clin Exp Med. 2015 Jan 15; 8(1):1286-90.

12. Rotterdam ESHRE/ASRM-Sponsored PCOS Consensus Workshop Group. Revised 2003 consensus on diagnostic criteria and long-term health risks related to polycystic ovary syndrome. Fertil Steril 2004; 81:19-25.

13. World Health Organization. Laboratory manual of the World Health Organization for the examination of human semen and spermcervical mucus interaction. Ann $\mathrm{I}^{\text {st }}$ Super Sanita 2001; 37(I-XII):1-123.

14. Takasaki A, Tamura H, Miwa I et al. Endometrial growth and uterine blood flow: a pilot study for improving endometrial thickness in the patients with a thin endometrium. Fertil Steril. 2010; 93(6):1851-8.

15. Nagori C, Panchal S. Endometrial vascularity: its relation to implantation rates. Int $\mathrm{J}$ Infertil Fetal Med. 2012; 3:48-50.

16. Molina A16-Sánchez J, Sánchez W, Vielma V. Platelet-rich plasma as an adjuvant in the endometrial preparation of patients with refractory endometrium. JBRA Assist Reprod. 2018 Mar 1; 22(1):42-48.

17. Casper RF. It's time to pay attention to the endometrium. Fertil Steril. 2011;96(3):519-21.

18. Hofmann GE, Thie J, Scott Jr RT, Navot D. Endometrial thickness but not pattern determined by transvaginal ultrasound is predictive of histologic evaluation of endometrial maturation 
in women undergoing hormone replacement for ovum donation. Fertil Steril 1996; 66:380-3.

19. El-Toukhy T, Coomarasamy A, Khairy $M$ et al (2008) The relationship between endometrial thickness and outcome of medicated frozen embryo replacement cycles. Fertil Steril 89:832-839

20. Samy, A., Abbas, A.M., Elmoursi, A. et al. Effect of autologous platelet-rich plasma transfusion in the treatment of infertile women with thin endometrium and its implications in IVF cycles: a literature review. Middle East Fertil Soc J 25, 5 (2020). https://doi.org/10.1186/s43043-020-0019-5

21. Sipahi M. Effects of Autologous PlateletRich Plasma on Endometrium Thickness and Pregnancy Rates During Intrauterine Insemination. Middle Black Sea Journal of Health Science, 2019; 5(1): 63-66

22. Tandulwadkar SR, Naralkar MV, Surana AD,
Selvakarthick M, Kharat AH. Autologous intrauterine platelet-rich plasma instillation for suboptimal endometrium in frozen embryo transfer cycles: A pilot study. J Hum Reprod Sci 2017;10:208-12.

23. Eftekhar M, Neghab N, Naghshineh E, Khani P (2018) Can autologous platelet rich plasma expand endometrial thickness and improve pregnancy rate during frozen-thawed embryo transfer cycle? A randomized clinical trial. Taiwanese $\mathrm{J}$ Obstet Gynecol. 57:810-813

24. Chang Y, Li J, Wei L-n, Pang J, Chen J, Liang $\mathrm{X}$. Autologous platelet-rich plasma infusion improves clinical pregnancy rate in frozen embryo transfer cycles for women with thin endometrium. Medicine 2019; 98.

25. Colombo GV, Fanton $\mathrm{V}$, Sosa $\mathrm{D}$, et al. Use of platelet rich plasma in human infertility. J Biol Regul Homeost Agents 2017; 31(2 Suppl. 2):179-182. 